\title{
Aerodynamic and Aeroacoustic Performance of Tail Rotor Investigation and Modification
}

\author{
Ai-Peng Hao ${ }^{1 *}$, Yu-Hong Jia ${ }^{2}$ \\ 1 School of Aeronautic Science and Engineering, Beihang University, Beijing, China; by1305108@buaa.edu.cn \\ 2 School of Aeronautic Science and Engineering, Beihang University, Beijing, China; jiayuhong@buaa.edu.cn \\ * Correspondence: by1305108@buaa.edu.cn
}

\begin{abstract}
With the increasingly stringent airworthiness standards, the noise generated during the rotorcraft flight is gradually attracting people's attention. It widely operated helicopters at low altitudes because of their maneuverability. The way to reduce the noise caused by the complex airflow of the helicopter rotor system has progressively become a hot topic for researchers. Using a hybrid acoustic analysis method, this paper investigates the improvement of the noise and thrust of the helicopter's tail rotor through the tail rotor structural parameters. For the basic model, the turbulence simulation is performed using an incompressible detached eddy simulation (DES) method, and the Lighthill acoustic analog equation is calculated using the finite element method (FEM). We verified the accuracy of the method through wind tunnel tests. We chose a series of structural parameters for sound simulation and fluid simulation calculations. The results indicate that the modified tail rotor noise reduced by $16.5 \mathrm{dBA}$ and the total thrust increased by $19.9 \%$ from the prototype model. This work can enhance the duct tail rotor design to improve aerodynamic and aeroacoustic performance.
\end{abstract}

Keywords: helicopter, tail rotor, aeroacoustic, finite element method, computational fluid dynamic

\section{Introduction}

Over the past few years, with the development of the civil aviation industry, helicopters have been used extensively in low-level operations. With the increasingly stringent airworthiness standards of aviation authorities around the world, how to reduce noise pollution has become an important consideration in the design of helicopters[1]. There are three main sources of noise in helicopter operations: rotor noise, engine noise, and transmission system noise[2-5]. The tail rotor of the helicopter is in a very complex flow environment due to the strong airflow disturbance of the main rotor, which poses a great challenge to the researchers[6]. The ducted tail rotor received considerable attention from helicopter designers for its compact size and high efficiency. During operation, the duct tail rotor has strong noise radiation due to the complex turbulence phenomena such as the high-speed blades constantly impacting the incoming flow at the duct inlet and the turbulent flow impacting the downstream stator blades[7]. The tail rotor of a helicopter is an important source of noise to passengers inside the cabin and residents around the airport. This is due to the proximity of the tail rotor to the cabin and the high-frequency noise generated by the tail rotor rotating at high speed, which is highly penetrating and difficult to be attenuated by the atmosphere during propagation[8]. Consequently, how to anticipate and reduce the noise from the helicopter's tail rotor is very important in practice.

In recent years, there has been extensive research on how to predict rotor noise and noise abatement methods[9]. To better understand the causes of noise generation and how to reduce effectively noise in rotor systems, researchers have carried out extensive research work, including theoretical analysis, numerical simulations, and experimental tests. These research efforts produced important results and provided a foundation for subsequent work. Farassat extended the Kirchhoff formulation to derive the mobile wall acoustic radiation models Farassat 1 and Farassat 1A. This model was used to calculate the noise at the distance of the helicopter's main rotor[10,11]. Ronald 
proposed an enhanced method for calculating turbulence noise and the helicopter rotor frequency spectrum. The method was used to analyze the noise and spread of the helicopter's main rotor system[12]. Lowson theoretically analyzed the noise model of the helicopter rotor, and in the theoretical analysis, the effects of propeller impact, rotational noise, and vortex noise on the noise of the rotor system were considered[13]. Song proposed an improved acoustic boundary element method and applied it to study the effect of moving sound sources on the design and surface of walls on sound propagation[14]. Shi introduced an active method of controlling rotor thickness noise. By adding a spoiler to the trailing edge of the rotor, they control the airflow separation on the wing surface to reduce the impact of upstream turbulence on the downstream propeller, thus reducing the overall rotor noise[5].

Compared to conventional helicopter tail rotors, ducted tail rotors have greater aerodynamic efficiency, smaller size, and higher safety. Because of the sheathing effect, the aerodynamic and aeroacoustic feature of the channeled tail rotor differs from that of the conventional rotor[15]. Cao established a numerical method to analyze the flow field in detail. They adopted CFD to calculate the flow field and tail rotor performance for both hover and lateral flight conditions. Many researchers are interested in the flow field and the location of the vortex core under different flight conditions[1618]. The prediction method is the key factor for ducted rotor noise analysis. The conventional algorithm uses CFD results combine with FW-H to calculate far-field noise radiation[19,20]. The finite element method has long been used in fluid-structure interaction issues, while the aeroacoustic finite element method is a recent development[9,21,22].

The main work of this paper is to introduce a hybrid acoustic analogy analysis method and apply it to discuss the effects of structural parameter variations on the thrust and noise reduction capability of the helicopter tail rotor system. We structure the content of this document along the following lines. The first part is an introduction. Section 2 introduces a hybrid acoustic analog methodology. To begin with, a computational model of fluid dynamics is introduced. Second, we introduced the method of nodal data transfer among different mesh densities. Lastly, the noise source equations for rotor systems are presented. Section 3 describes the structural parameters and mesh system of a helicopter tail rotor. We checked the precision of the numerical prediction method against the experimental results of the wind tunnel. Section 4 discusses the effects of some parameters on the helicopter tail rotor in terms of thrust and noise reduction. This paper concludes with Section 5.

\section{Methods}

Turbulence generated by the high-speed rotating propellers, turbulence affecting the downstream propellers, and pressure pulses on the inner wall of the diffuser primarily generated the helicopter tail rotor noise.

It divides the hybrid method of sound analogy described in this article into three major parts.

We consider thick noise the majority of rotor noise. First, the CFD method is used to calculate the pressure pulsation of the blade and the inner wall of the diffuser, and we derive the turbulence information of the grid nodes in CGNS format. Simultaneously, the tail rotor thrust is obtained.

The computational fluid dynamics grid and computational aeroacoustic have various grid densities. In the second stage, we transfer the turbulence information about the CFD grid nodes to the acoustic grid nodes using the data transfer algorithm.

Finally, Lighthill's acoustic analog equation is solved using the finite element method. By coordinated transformation, the acoustic model of the fan is obtained, and the noise of the ducted tail rotor in the far-field is calculated.

\subsection{Numerical solver for unsteady turbulent flow filed}

To demonstrate the turbulent flow mechanism, a commercial solver was used to simulate the flow field. Since most of the flow field around the helicopter rotor blades is considerably incompressible, except for the area close to the blade surface, the Navier-Stokes incompressible algorithm is used. The incompressible solver supplied by ANSYS Fluent is used and the governing equations are closed by the two-equation SST k-omega turbulence pattern[23]. The discretization of 
the diffusive and convective terms of the governing equations is a second-order precision. The dimension-free time step is chosen for each analysis individually, to obtain a Courant-FriedrichsLewy (CFL) number lower than 1 which is required by the calculation aeroacoustic solution algorithm.

\subsection{Data transfer strategy}

The hybrid acoustic analog calculation method introduced in this paper requires a data transfer method to accommodate the node information transfer for different grid densities because of the different densities of the fluid and acoustic grids. This data transfer method is referred to as the maximum range algorithm. It can be applied to transfer data between two sets of grids having the same form but a different number of grid nodes. So that the turbulence information on all nodes can be mapped from the fine CFD grid (source grid) to the coarse acoustic grid (target grid) and ensure the accuracy of the node data. This method is constructed using the Lobatto function. This function is a function of the size of the acoustic grid concerning the frequency to modify the order of the grid. This strategy enables the application of a coarse acoustic mesh over the whole frequency range. It could increase the order of the coarsest element at high frequencies and small acoustic wavelengths locally. Figure 1 shows an example of data transfer between two sets of bidimensional grids.

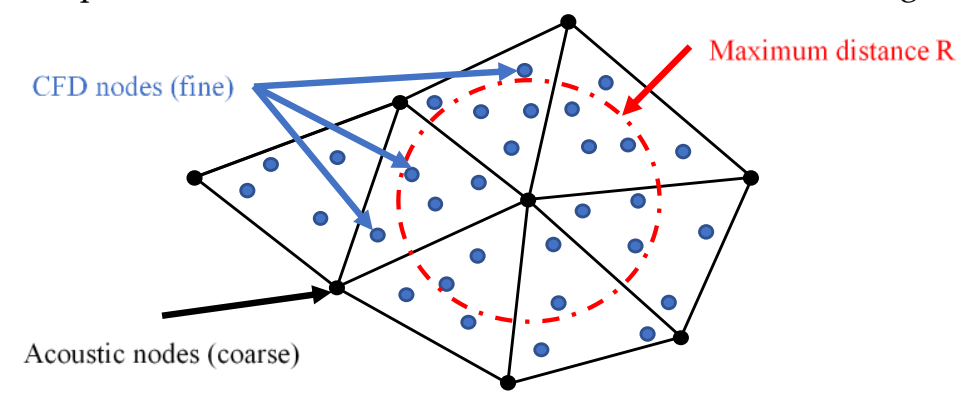

Figure 1. Example of 2-D grid data transfer.

The method has two important settings. The first parameter refers to the number of grid nodes $\mathrm{N}$. If the maximum number of nodes is below the actual number of nodes in the CFD grid, the average of the CFD grid is calculated. The latter is the maximum distance R. Only the CFD grid nodes located inside the maximum distance from the acoustic grid nodes are considered for data transfer.

The $\mathrm{N}$ nodes closest to the given CFD grid node are selected to transmit data to the acoustical grid node.

The target node data on the acoustic grid is calculated as a weighted mean of the source node data of the selected CFD grid by expression (1).

$$
W_{i}=\frac{1}{d_{i}} / \sum_{i=1}^{N} \frac{1}{d_{i}}
$$

The value transferred from the target node is defined as (2).

$$
P_{\text {acoustic }}=\sum_{i=1}^{N} \frac{P_{i}^{C F D}}{d_{i}} / \sum_{i=1}^{N} \frac{1}{d_{i}}
$$

Where $d_{i}$ represent the distance from the CFD grid to the acoustic grid. $P_{i}^{C F D}$ is the pressure value on the CFD grid.

After the acoustic mesh node data obtained from the source grid node, the inlet and diffuser region are discretized using the finite element method, and the Helmholtz equation is resolved. Due to the existence of complex structures such as blades, hub covers, and sidewalls on the inner surface of the duct, the automatic pairing layer (AML) is used as the radiation boundary of the external infinite far field when calculating the radiation from the sound source to the outside. At the same time, the finite grid limit causing acoustic pseudo-reflection is avoided[24]. The reflections and diffuser of arbitrary geometrical shapes can be taken into account so that the calculation allows to separate the intrinsic interference characteristics of the source and the presence of the shroud. The 
calculation does not consider the flow rate and is valid only when the axial Mach number is sufficiently small.

This method calculates the reflection and diffuser of sound waves from any surface separately. The calculation can distinguish between the interference of sound waves from the blade and the reflection of sound waves from the inner wall of the diffuser.

\subsection{Tonal nosie formulation}

The noise generated by helicopter rotor blades can be considered as consisting of broadband noise generated by random aerodynamic loads on the blade surface with pure tone noise generated by the incoming flow and inter-blade interaction.

According to the Farassat derivation [11], the sound of the rotor blades is divided into three main components as follows.

The first is the thickness noise, which is generated by the displacement of the pushing air of each blade.

The second is the load noise, which is generated by the interaction of the airflow with the blades. The third is quadrupole noise, which is generated by the vortex that flows into the rotor trail.

Here, we analyze for one rotor with uniformly distributed B blades. Due to the uniform blade distribution, only the blade passing frequency (BPF) harmonics are generated at this stage. Suppose the existence of a rotating network of dipoles distributed in a circle of radius $r$ with the origin of the reference system as the center (as shown in Figure 2). As each blade consists of one repeat of the same surface elements, the entire paddle may be reduced to a point source. The magnitude of the sound pressure generated by the BPF harmonics of the order of $m$ in the far-field can be extended to the sum of the radiation modes.

Supposing that the pallet is a thin blade, the thickness noise can be overlooked. Because the sound of the dipole is much bigger than that of the quadrupole, the sound of the quadrupole is also neglected. In the progress of research, it is assumed that the microphone is in the far-field and that the audio sources are compact. These sound sources are represented by a reference system of motion fixed on the blades, as illustrated in Figure 2.

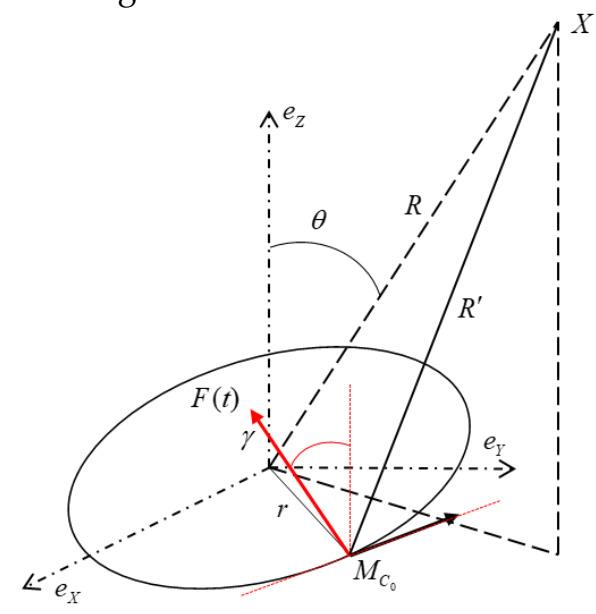

Figure 2. Reference frame for rotor-noise problem simulation configuration.

In this section, the duct rotor is considered a rotor-stator configuration[22]. Then, the axial and tangential contributions from the radiated pressure to the frequency $m B \Omega$ are expressed as (3).

$$
\begin{aligned}
\mathrm{p}_{\mathrm{inc}}^{\mathrm{mB}}(\overrightarrow{\mathrm{x}})= & \frac{-\mathrm{imBV} \Omega \mathrm{e}^{-\mathrm{imB} \Omega \mathrm{R} / \mathrm{c}_{0}}}{4 \pi \mathrm{c}_{0} \mathrm{R}} \mathrm{F}_{S} \sum_{\mathrm{s}=-\infty}^{\infty} \mathrm{e}^{-\mathrm{i}(\mathrm{mB}+\mathrm{sV})\left(\varphi+\frac{\pi}{2}-\alpha_{0}\right)} \\
& \times \mathrm{J}_{\mathrm{mB}+\mathrm{sV}}(-\mathrm{mBM} \sin \theta)\left[\cos \gamma^{\prime} \cos \theta-\frac{\mathrm{mB}+\mathrm{sV}}{\mathrm{mBM}} \sin \gamma^{\prime}\right]
\end{aligned}
$$


where the observer is located in a spherical coordinate system $(R, \theta, \varphi)$. The factors $F_{S}$ are the Fourier coefficients of the node dipole force on the grid, defined by the discrete Fourier transformation(4).

$$
F(t)=\sum_{S=-\infty}^{\infty} F_{S} e^{-i S \Omega t} \quad, \quad F_{S}=\frac{\Omega}{2 \pi} \int_{0}^{2 \pi / \Omega} F(t) e^{i S \Omega t} d t
$$

Equation (3) assumes that the dipole point source of the blade is on the axis $e_{X}$ at the original time. This equation calculates the total acoustic pressure of the rotor blade. The contribution of the sound of each blade element along the axial and tangential directions is corrected by the double summation of all elements on the discrete blades. We can compute acoustic radiation from sources at different locations at the same time. It explains the propagation path difference.

The formula for the tonal noise of the uniform blade stator under the same harmonic BPF caused by the regular impact of the blade turbulence with radius $r$ is obtained according to a similar principle. A blade is discrete in compact units if a discrete method is necessary, the same unit is used for each blade. Alternately, the blade is partitioned into plane units for analysis and modeling. For convenience, the origin of the reference system is moved to the center of the chord circle of the line array, using the same observer symbol. The origin of the terms of reference is moved to the center of the line segment network chord circle, with the same sign for the observer's contact information. This allows the rotor and stator radiation characteristics to be compared. The far-field sound pressure reading as (5).

$$
\begin{aligned}
\mathrm{p}_{\text {inc }}^{\mathrm{mB}}(\overrightarrow{\mathrm{x}})= & \frac{\mathrm{mBV} \Omega \mathrm{e}^{-\mathrm{imB} \Omega \mathrm{R} / \mathrm{c}_{0}}}{4 \pi \mathrm{c}_{0} \mathrm{R}} \mathrm{F}_{S} \sin \theta \\
& \times \sum_{\mathrm{s}=-\infty}^{\infty} \mathrm{e}^{-\mathrm{i}(\mathrm{mB}+\mathrm{sV})\left(\varphi+\frac{\pi}{2}-\alpha_{0}\right)} \mathrm{J}_{\mathrm{mB}+\mathrm{sV}}^{\prime}(-\mathrm{mBM} \sin \theta)
\end{aligned}
$$

where $\mathrm{m}$ is a harmonic number, $\mathrm{B}$ is the number of blades, $\Omega$ is rotational speed, $R_{i}$ is the distance from the observer to the origin of the specified axis system, $c_{0}$ is sound speed, $\mathrm{M}$ is rotational Mach number, $\theta$ and $\gamma$ are defined in Figure 2, $\mathrm{V}$ is the number of stator blades. For the duct tail rotor studied in this paper, $\mathrm{V}$ is set equal to 1 , i.e., there is no blade exit.

\section{Modeling and Validation of the Methodology}

\subsection{Prototype of helicopter tail rotor structure}

In this section, a basic helicopter duct tail rotor model, shown in Figure 3, is presented for wind tunnel testing and validation of the numerical method. Due to the weak influence of the fin on the thrust and noise of the tail rotor, the geometric model ignores the helicopter fin during the numerical analysis. 


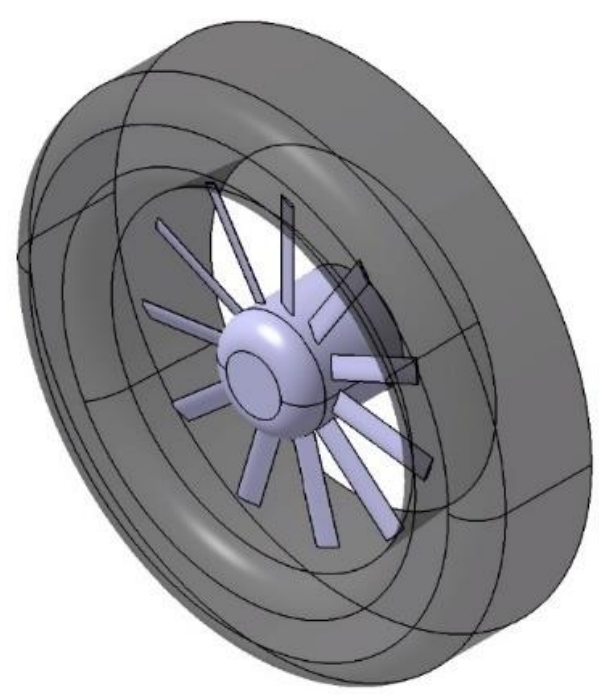

Figure 3. Diagram of the construction of the duct tail rotor.

The main specifications of the duct tail rotor are set out in Table 1 . The structure is shown in Figure 4.

Table 1. Main specification for the prototype rotor system.

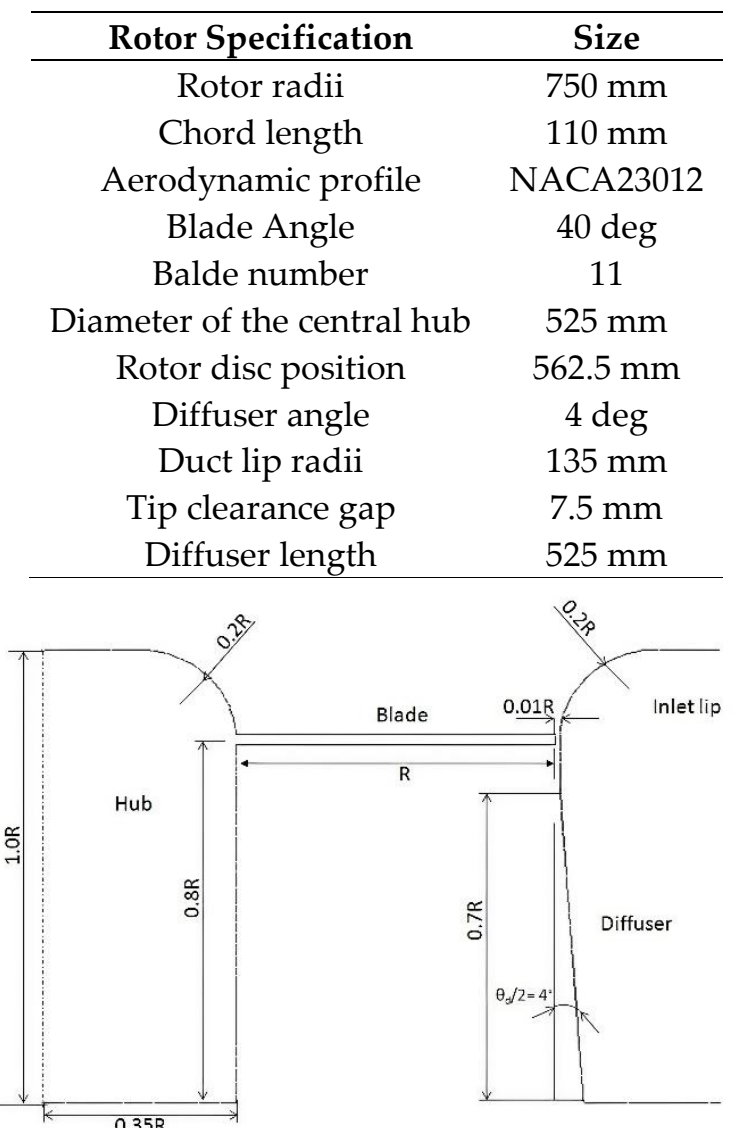

Figure 4. Cross-section of the fairing and hub at the midpoint of the azimuth of the duct tail rotor.

\subsection{Mesh generation}

For the tail rotor aerodynamic analysis, the complete flow field is split into two parts.

1. The inlet port and the rotating region where the rotor is located.

2. The stationary area of the diffuser section of the duct. 
The area interface for the rotating reference frame is illustrated in Figure 5. Note that the area of the rotating reference frame extends to the entrance of the inlet lip. This may seem counterintuitive, as the hub cone and input lip are at the same height, so the flow does not revolve in this area. The stationary field consists of the part of the diffuser fairing and the center hub. The flow field is entangled in an unstructured grid. The grids for shroud surface, hub surface, and blade surface are presented in Figure 6. In the area of the leading and trailing edges of the blade, an encrypted grid was used and the boundary layer cells were divided. In the clearance area between the tip and the inner wall of the duct, the grid was encrypted, as shown in Figure 7.. Depending on the input and output regions, the total cell count for the entire flow field is approximately 18.5 million. There are 3.6 million rotor cells and 1.1 million sheath cells.

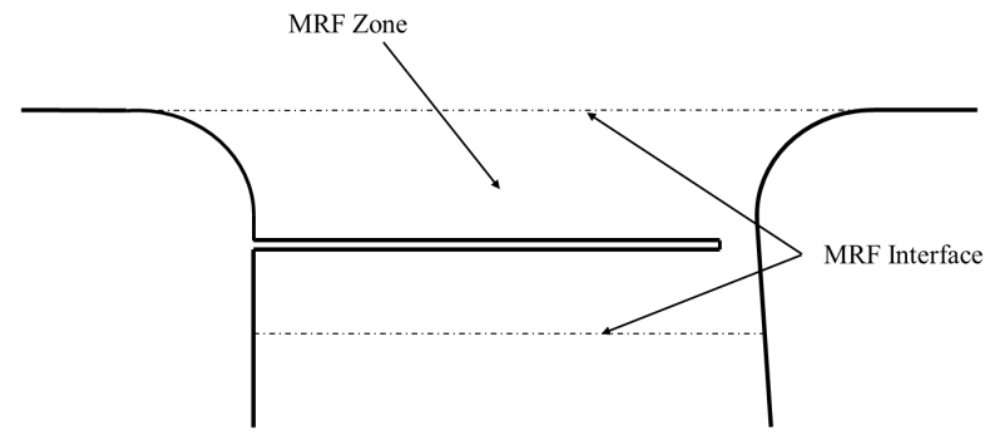

Figure 5. MRF zone in CFD mesh.

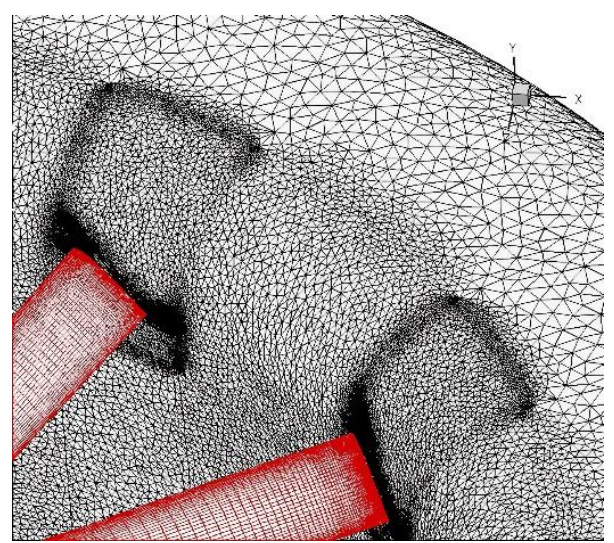

(a)

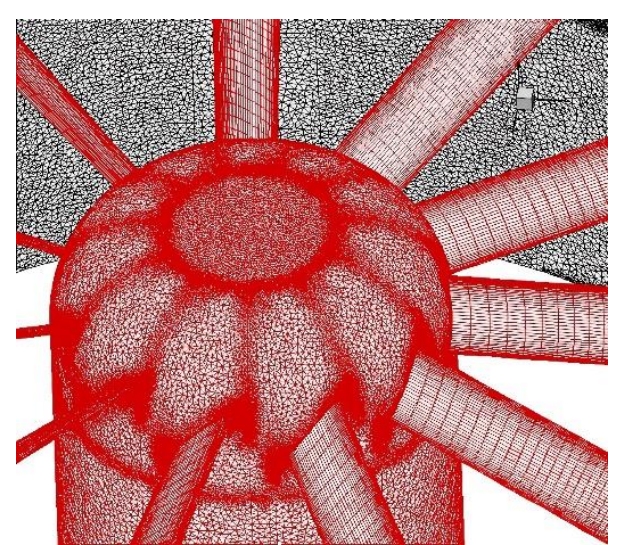

(b)

Figure 6. Unstructured grid for duct tail rotor surface: (a) inlet lip and diffuser surface mesh; (b) hub and blade surface mesh.

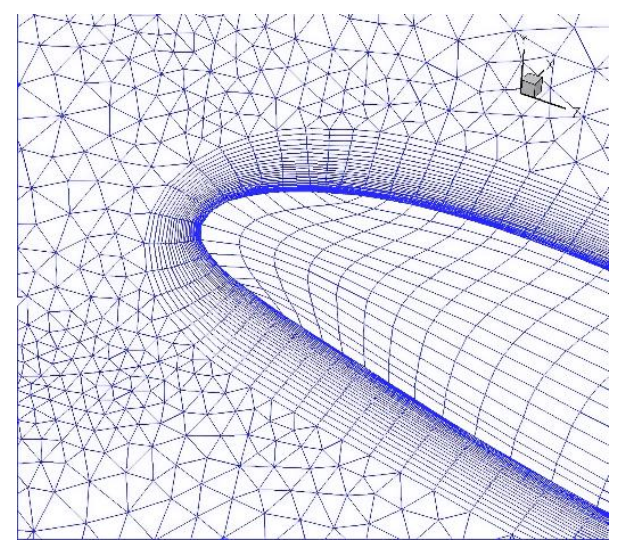

(a)

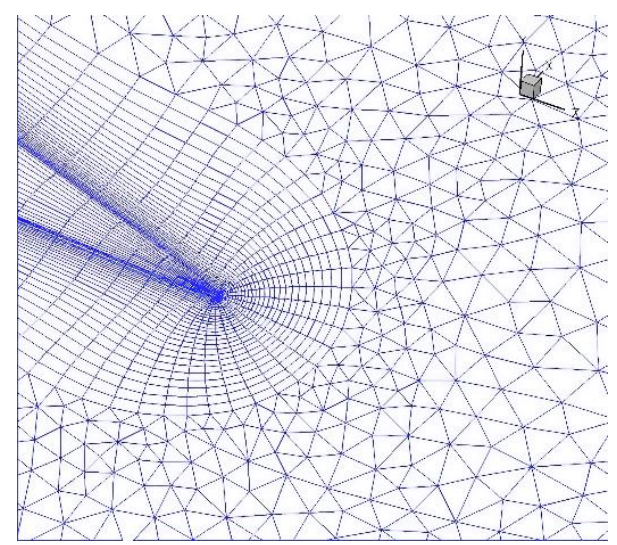

(b) 
Figure 7. Mesh of the boundary layer of a single blade and its projection on the diffuser wall: (a) Trailing edge mesh; (b) Leading edge mesh.

The acoustic mesh used in this document is illustrated in Figure 8 and comprises 15000 nodes 106000 triangular elements, as shown in Figure 8. The highest acoustic frequency that can be solved in a conventional finite element solver with the one order grid is $740 \mathrm{~Hz}$, according to the relationship between the acoustic wavelength that needs to be satisfied and the grid size, as expressed in the equation (6). In this paper, we used the Adaptive Finite Element Method for Resolution (FEMAO) [25]. The solver automatically adjusts the sequence of the local grid according to the frequency of interest and the location of the sound source.

$$
f=\frac{c(1-M)}{L}
$$

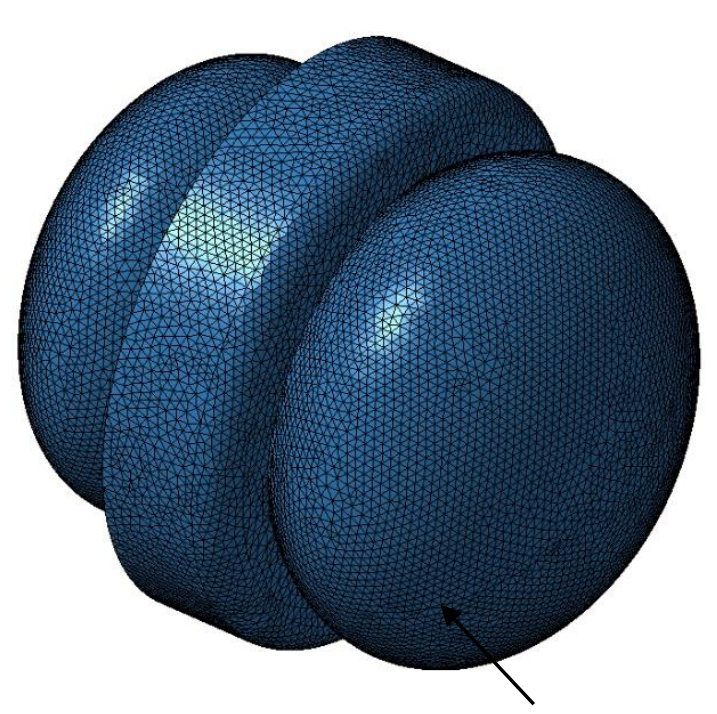

AML surface for acoustic propagation

Figure 8. Acoustic grid for FEMAO acoustic analogy.

\subsection{Results}

\subsubsection{Wind tunnel facilities}

To verify the accuracy of the hybrid acoustic analog, acoustic wind tunnel measurements of the duct tail rotor were performed in the low-speed aeroacoustic wind tunnel FL-17 at the China Aerodynamics Research and Development Center, as illustrated in Figure 9. The background noise of the wind tunnel in the open test section was $75.6 \mathrm{dBA}$ (at $7.95 \mathrm{~m}$ from the nozzle centerline, $80 \mathrm{~m} / \mathrm{s}$ wind speed). Considering the complex airflow interference between the main rotor and tail rotor, and the effect of the wash airflow under the main rotor on sound propagation, only the thrust and noise of the tail rotor are tested in the wind tunnel experiment. The microphone array was arranged at $5 \mathrm{~m}$ from the center of the tail rotor, and the blade tip Mach number was 0.23. 


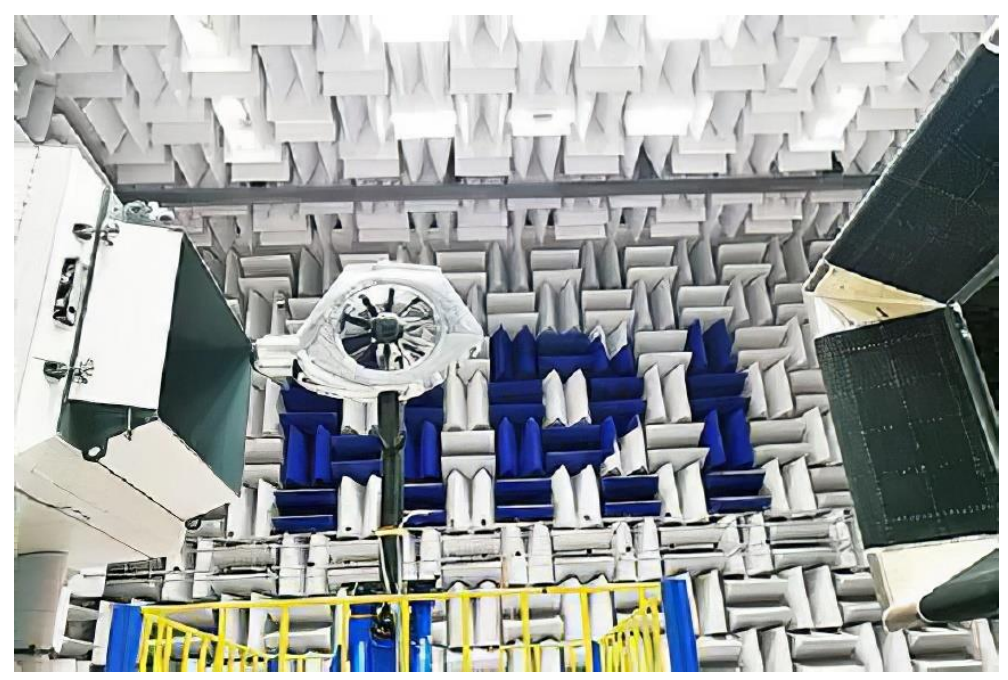

Figure 9. Helicopter duct tail experiment in aeroacoustic wind tunnel FL-17 at CARDC.

\subsubsection{Flow field simulation results}

Figure 10 shows the pressure distribution at the fairing and blade surface. As indicated by Figure 10 (b), the pressure in the duct inlet produced a drastic change, while the air pressure remained constant in the diffuser section. Pressure imbalance in the inside wall of the duct before and after the rotor disk is the main reason for the push into the duct. Besides, the conclusion can be achieved from the rotor thrust is mostly generated by the rotor tip and the inlet lip.

The midpoint plane of the azimuth is widely used in channeled tail rotor aerodynamics documentation, to describe the flow distribution. Figure 11 shows the total flow distribution and local distribution away from the end of the blade near the lintel wall. It can be seen that the overall distribution of the flow is consistent within and through the rotor in terms of flow uptake, consistent with the duct being in hovering flight. The rotor receives a mass flow in all directions, without obvious areas of re-circulation.

A comparison of the speed distribution along with the axial directions and the application of the duct tail rotor in experimental trials and numerical simulations is presented in Figure 12. The predictions are generally consistent with the results of the experience.
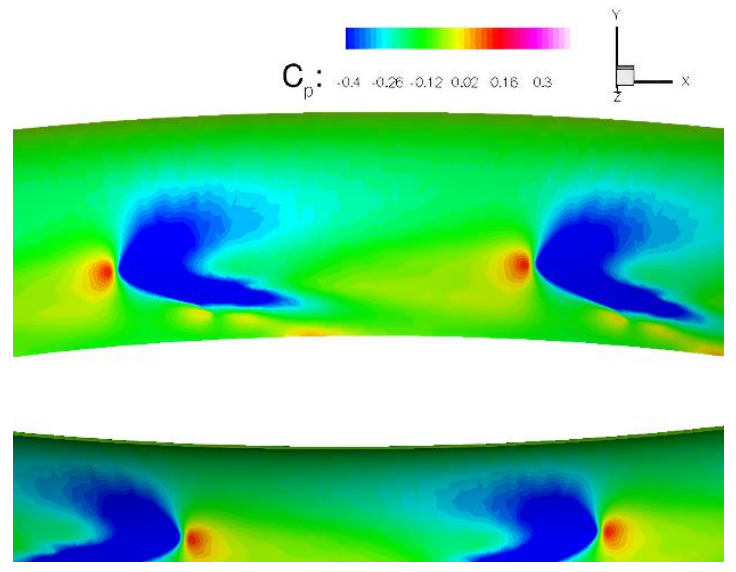

(a)

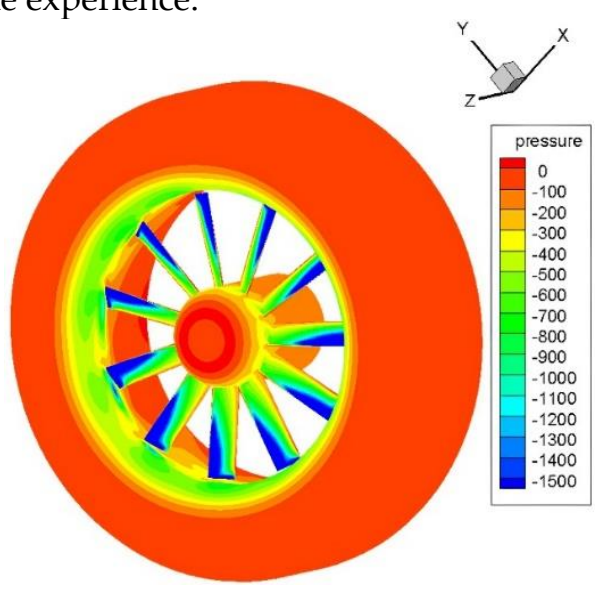

(b)

Figure 10. Surface pressure contours for duct tail rotor: (a) inlet lip pressure contour distribution; (b) Portside surface pressure. 


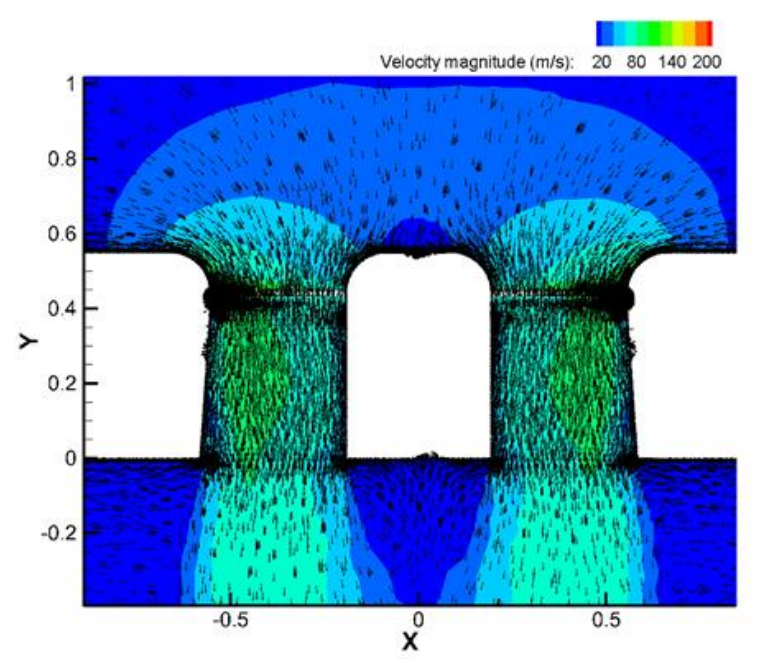

(a)

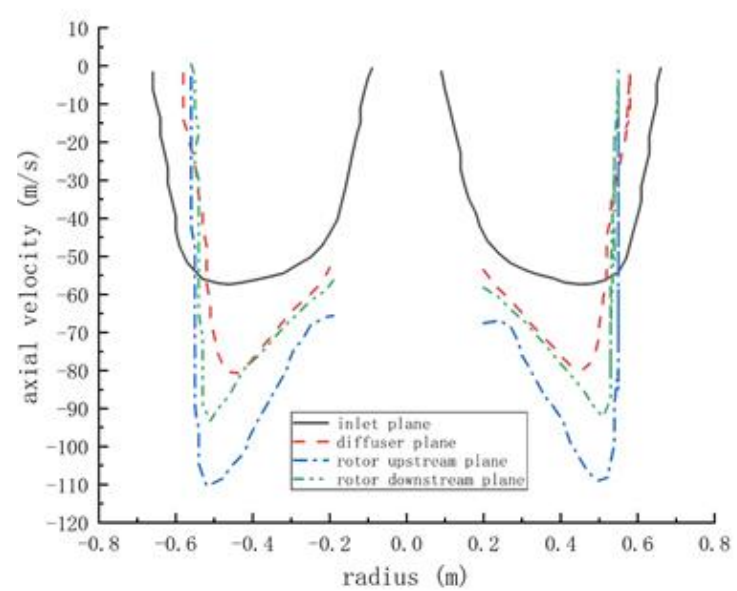

(b)

Figure 11. Velocity distribution in rotor: (a) velocity vector at mid-azimuth plane; (b) Axial speed distributions on different parts of the tail rotor.

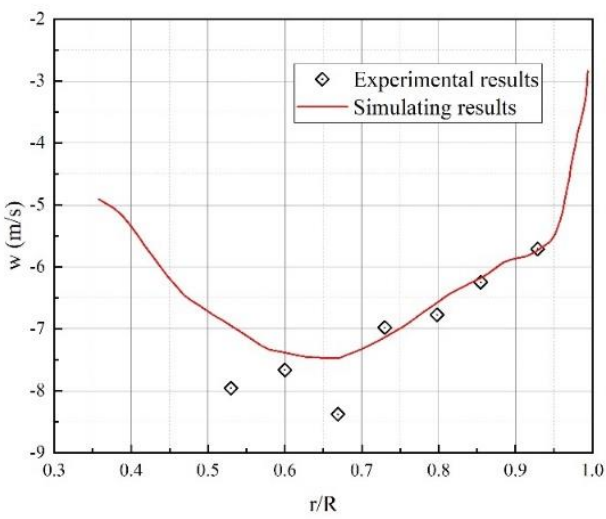

(a)

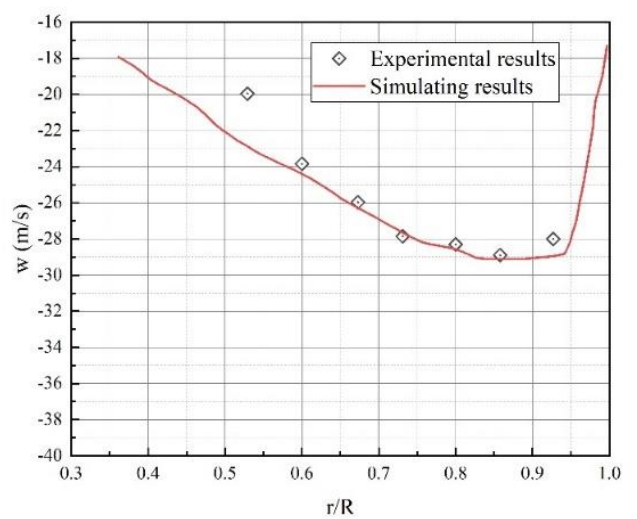

(b)

Figure 12. Comparison of the speed distribution between experiment and numerical simulation: (a) extended distribution of induced rejections; (b) extended distribution of circumferential induced velocity.

\subsubsection{CAA results comparison with wind tunnel test}

Acoustic radiation signals from the helicopter ducts are sampled and processed by the microphones system. Figure 13 shows the comparison of experimental and computed sound pressure levels with 6 harmonic numbers. It is possible to observe that the numerical prediction method has the same tendency as the experimental measures. The blade passage frequency (BPF) is calculated by equation (7).

$$
B R F=\frac{N \times R}{60}
$$

where $\mathrm{N}$ denotes the rotor speed and $\mathrm{R}$ denotes the rotor radius. In this case, the rotating speed is $2310 \mathrm{rpm}$, and the blade radius is $750 \mathrm{~mm}$, the corresponding BPF is $481 \mathrm{~Hz}$. 


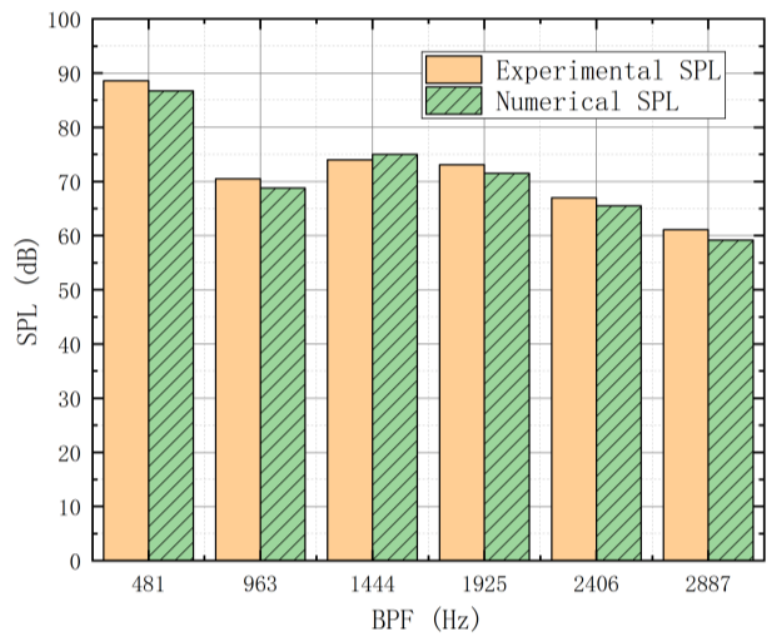

Figure 13. Comparison of wind tunnel investigational results with numerical results.

\section{Different effects of the parameters on the performance of the Fenestron tail rotor}

In this section, we examine the effect of tail rotor parameters on noise and thrust using the hybrid acoustic analogical method described above. We discuss some parameters, including the aerodynamic profile of the blade, the chord length of the blade, the angle of the blade, the angle of the diffuser, the clearance of the tip, and the radius of the inlet.

\subsection{Effect of airfoil change on tail rotor thrust and noise performance}

Based on a prototype of duct tail rotor, we investigated the effect of variation of the blade airfoil type on the tail rotor in terms of thrust and aeroacoustic. Four typical airfoils of the tail rotor were selected for investigation. The thrust and noise generated by different types of airfoils are shown in Table 2, and the characteristics of far-field noise directivity are shown in Figure 14.

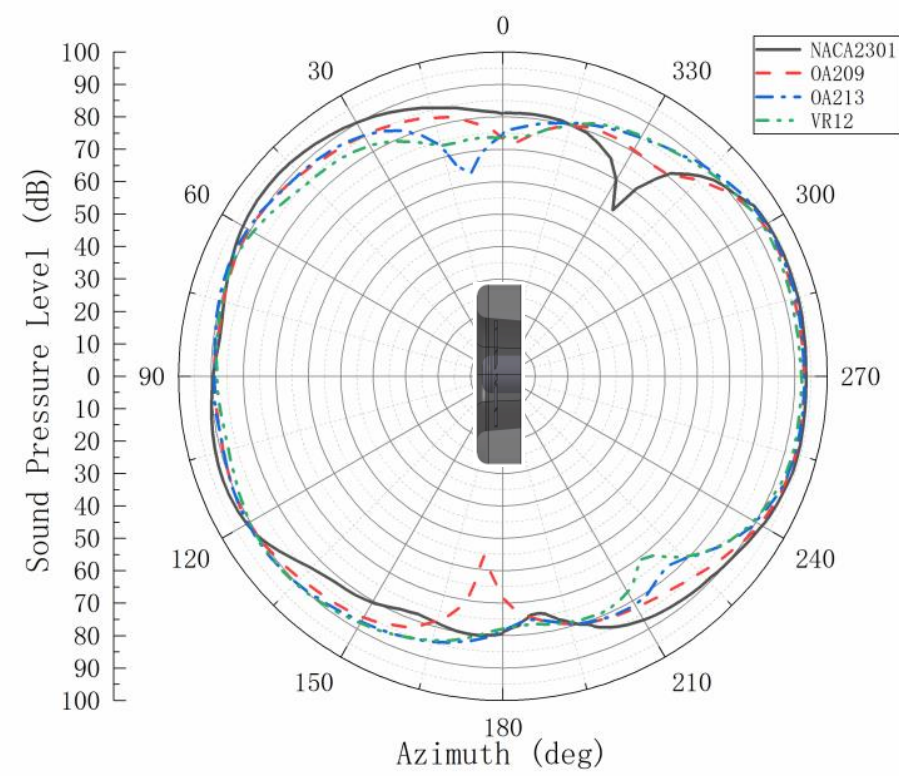

Figure 14. Far-field sound directional features of different types of airfoils.

Table 2. Blade aerodynamic profile modification tasks and corresponding outcomes.

\begin{tabular}{ccc}
\hline Airfoils & OASPL(dB) & Thrust(N) \\
\hline
\end{tabular}




\begin{tabular}{cll}
\hline NACA23012 & 86.7 & 6244.7 \\
OA-209 & 85.3 & 6473.1 \\
OA-213 & 86.0 & 6538.8 \\
VR-12 & 85.1 & 6319.5 \\
\hline
\end{tabular}

In this section, only the modification of the blade's aerodynamic profile is taken into account. The global far-field sound pressure levels for each of the four airfoil types are $86.7 \mathrm{dBA}, 85.3 \mathrm{dBA}$, $86.0 \mathrm{dBA}$, and $85.1 \mathrm{dBA}$, respectively. We note that the change in the type of aerodynamic profile does not significantly affect the thrust and overall sound pressure level of the field away from the reformed tail rotor. By examining the flow field information, we found that the change of airfoil did not significantly change the streamline of the duct airflow and the distribution of vortices, which in turn led to similar turbulence structures. According to the calculation results, the airfoil with the lowest overall sound pressure level among the four airfoils is VR-12, while the corresponding thrust is range in the middle. In subsequent calculations, we use the VR-12 as a basis to further investigate the effects of structural parameters on the thrust and sound of the reformed duct tail rotor.

\subsection{Effect of chord length change on tail rotor thrust and noise performance}

In this section, we use the base model of the VR-12 airfoil to study the effect of the variation of the blade chord length on the helicopter tail rotor in terms of thrust and aeroacoustics, respectively. In this case, four cord lengths are selected for the study. Thrust and noise for different lengths are shown in Table 3, and the directionality characteristics of far-field noise are shown in Figure 15.

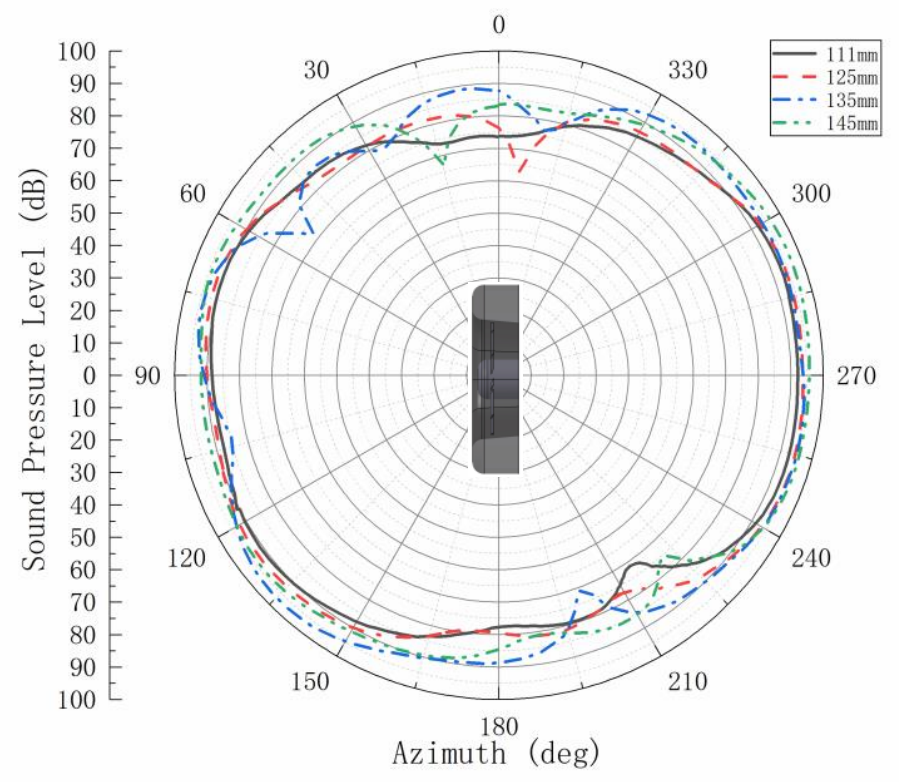

Figure 15. Far-field sound directional features of different length of blade chord.

Table 3. Chord length change tasks and associated results.

\begin{tabular}{ccc}
\hline Chord length & OASPL(dB) & Thrust(N) \\
\hline $111 \mathrm{~mm}$ & 85.1 & 6319.5 \\
$125 \mathrm{~mm}$ & 86.7 & 6600.6 \\
$135 \mathrm{~mm}$ & 89.2 & 6669.8 \\
$145 \mathrm{~mm}$ & 89.5 & 7081.3
\end{tabular}

The overall sound pressure levels corresponding to the four chord lengths are $85.1 \mathrm{dBA}, 86.7$ $\mathrm{dBA}, 89.2 \mathrm{dBA}$, and $89.5 \mathrm{dBA}$, respectively. The thrust and noise of the reformed tail rotor show the same increasing tendency with the length of the blade chord increasing. We found that the increased length of the chord significantly altered the pressure distribution in the area near the rotor disc and affected the distribution of the vortices downstream. This results in a change of the dipolar sound 
source at the internal surface of the diffuser section. Therefore, the increase in blade chord length has an important effect on the performance of the helicopter's tail rotor in terms of thrust and acoustics.

\subsection{Effect of blade angle change on tail rotor thrust and noise performance}

In this section, we consider the impact of blade angle of attack on thrust and noise production. We discuss the variable angle of attack of the rotor blades between 40 degree and 50 degree. In the initial design, the rotor blade is installed at a 40 degree. Table 4 below shows the calculated total sound pressure and thrust results for different blade angles. The directionality characteristics of farfield sound are illustrated in Figure 16.

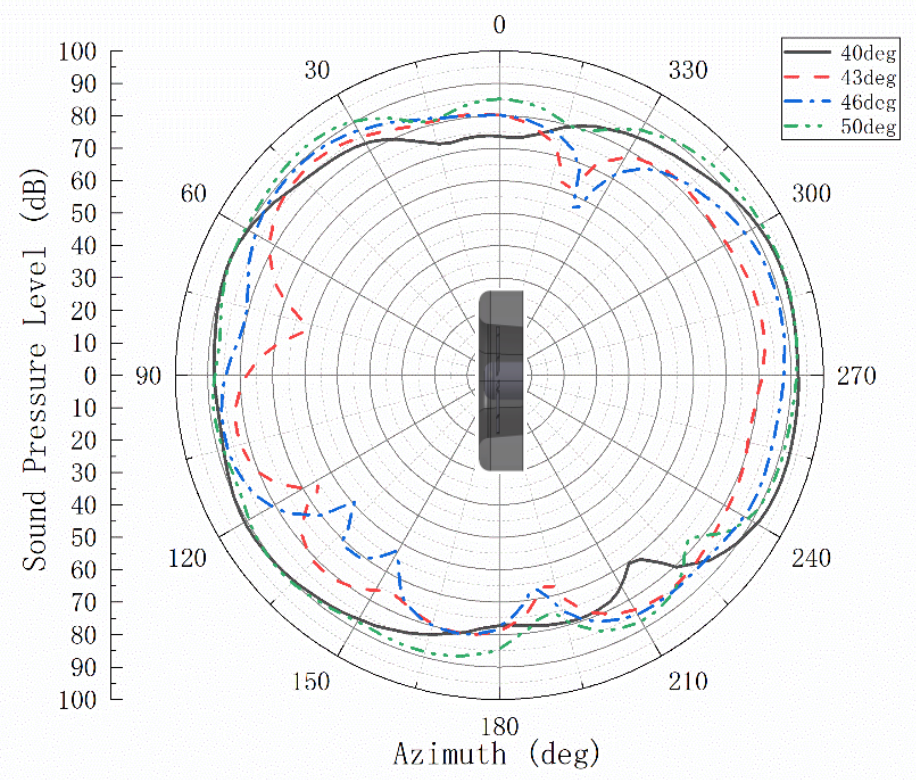

Figure 16. Far-field sound directional features of different blade angle.

Table 4. Blade angle change tasks and associate results.

\begin{tabular}{ccc}
\hline Blade angle & OASPL(dB) & Thrust(N) \\
\hline $40 \mathrm{deg}$ & 85.1 & 6319.5 \\
$43 \mathrm{deg}$ & 79.2 & 7536.8 \\
$46 \mathrm{deg}$ & 81.5 & 7225.0 \\
$50 \mathrm{deg}$ & 87.7 & 6647.5 \\
\hline
\end{tabular}

The overall sound pressure levels corresponding to the four-blade angles of attack are $85.1 \mathrm{dBA}$, $79.2 \mathrm{dBA}, 81.5 \mathrm{dBA}$, and $87.7 \mathrm{dBA}$, respectively. As shown in Table 4, the overall thrust of the helicopter's tail rotor increased as the blade angle increased between $40 \mathrm{deg}$ and $43 \mathrm{deg}$. However, as the blade angle continues to increase to 50 degrees, the total thrust of the tail rotor gradually decreases. We found that as the blade angle of attack increased, the blade showed a local stall phenomenon, and the airflow on the blade surface separated, which would reduce the pressure difference between the inlet and the diffuser section and create strong turbulence, resulting in increased sound pressure in the far-field. Figure 15 shows that the noise pointing characteristics of the duct wake blade are significantly lower than the other three angle of attack settings when the blade angle of attack is 43 degrees. Consequently, an appropriate adjustment of the blade angle of attack may reduce the aerodynamic noise of the helicopter's tail rotor while increasing thrust.

\subsection{Effect of diffuser angle change on tail rotor thrust and noise performance}

In this section, we examine the effect of change in diffuser angle on thrust and noise. We discuss the effect of the angle change of the diffuser angle of the duct within the range of 4 degree to 5.5 degree. The original design is designed with a 4 degree diffuser angle. Table 5 shows the overall 
sound pressure level and the thrust results corresponding to the various diffuser angles. The directionality characteristics of far-field noise are illustrated in Figure 17.

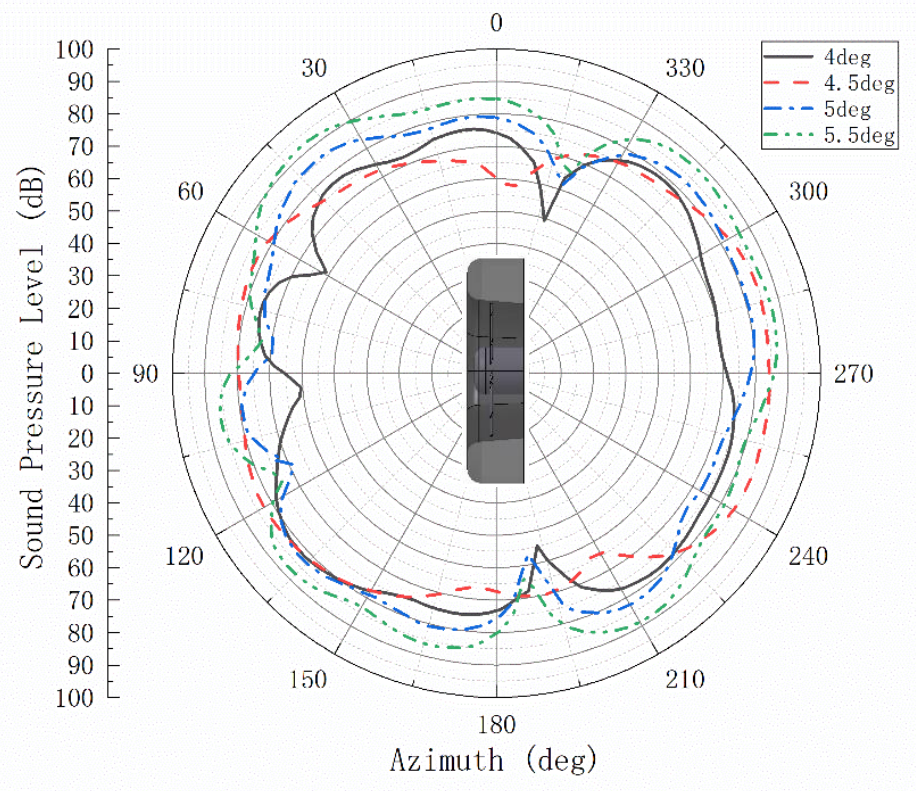

Figure 17. Far-field sound directional features of different length of diffuser angle.

Table 5. Diffuser angle change tasks and associate results.

\begin{tabular}{ccc}
\hline Diffuser angle & OASPL(dB) & Thrust(N) \\
\hline 4 deg & 70.2 & 7487.6 \\
$4.5 \mathrm{deg}$ & 72.9 & 7416.6 \\
$5 \mathrm{deg}$ & 74.3 & 7433.0 \\
$5.5 \mathrm{deg}$ & 79.7 & 9955.9 \\
\hline
\end{tabular}

The total sound pressure levels for the four diffuser angles of the duct are $70.2 \mathrm{dBA}, 72.9 \mathrm{dBA}$, $74.3 \mathrm{dBA}$, and $79.7 \mathrm{dBA}$, respectively. In Table 5, it can be seen that the total sound pressure in the far-field of the helicopter increases gently first with the increase of the diffuser angle, and the tail rotor noise increases significantly when the diffuser angle increases to 5.5 degree. The helicopter thrust also exhibits a similar trend with the change in the diffuser angle of the duct. This is since as the diffuser angle increases to some extent, the clogging effect of the airflow in the diffuser section is considerably reduced. The dynamic pressure in the diffuser section is reduced and the airflow is increased. In the 4 diffuser angle settings above, the original diffuser angle of 4 degree has the lowest overall sound pressure in the far-field. When designing the diffuser angle of the duct, the range for selecting the diffuser angle should be studied from the point of view of thrust and noise.

\subsection{Effect of tip clearance change on tail rotor thrust and noise performance}

In this section, we look at the effect of variation of the blade tip clearance on thrust and noise. We discuss the effect of the variation of the clearance between $7.5 \mathrm{~mm}$ and $18.75 \mathrm{~mm}$ in the blade tip clearance range. Table 6 presents the overall sound pressure and thrust results for the various blade tip releases. The directionality characteristics of far-field sound are illustrated in Figure 18. 


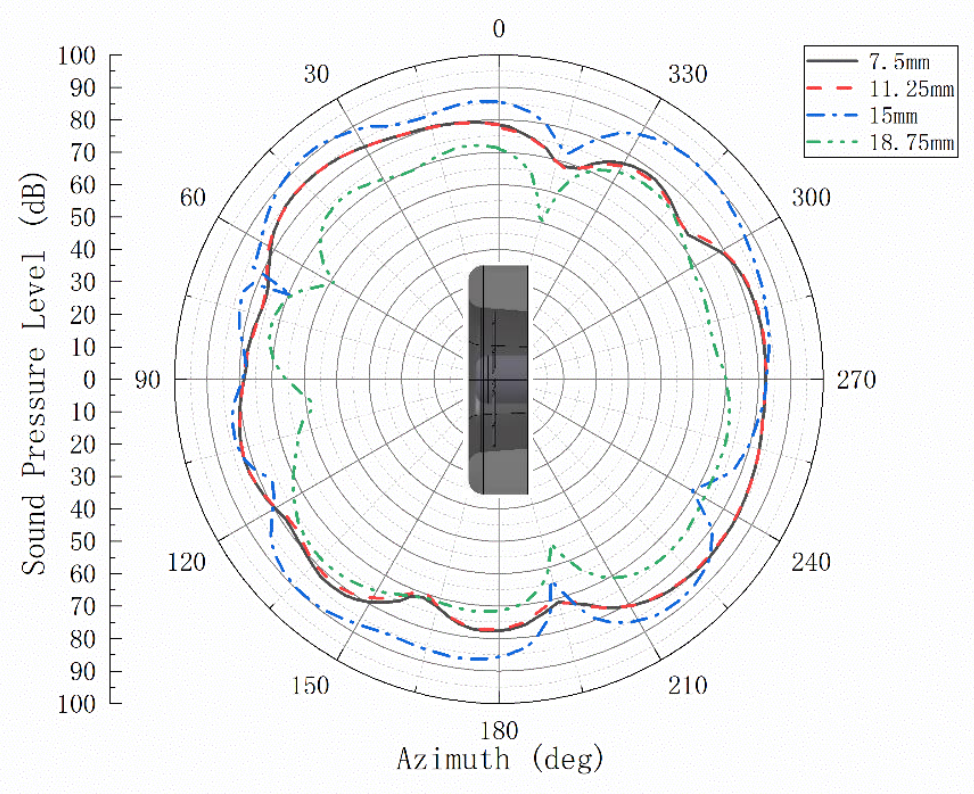

Figure 18. Far-field sound directional features of different lengths of tip clearance.

Table 6. Tip clearances change tasks and associate results.

\begin{tabular}{ccc}
\hline Tip clearance & OASPL(dB) & Thrust(N) \\
\hline $7.5 \mathrm{~mm}$ & 79.5 & 8788.8 \\
$11.25 \mathrm{~mm}$ & 79.2 & 8789.3 \\
$15 \mathrm{~mm}$ & 84.1 & 9478.4 \\
$18.75 \mathrm{~mm}$ & 70.2 & 7487.6 \\
\hline
\end{tabular}

The overall sound pressure levels for the four tip clearances are $79.5 \mathrm{dBA}, 79.2 \mathrm{dBA}, 84.1 \mathrm{dBA}$, and $70.2 \mathrm{dBA}$, respectively, and the thrust and noise levels are almost the same at $7.5 \mathrm{~mm}$ and 11.25 $\mathrm{mm}$. When the tip clearance is $15 \mathrm{~mm}$, the thrust and overall sound pressure level of the tail rotor is at the highest of the four settings. However, when the tip clearance is increased to $18.75 \mathrm{~mm}$, the thrust and noise of the tail rotor are reduced to the lowest of the four settings. Since when the tip clearance is low, the airflow close to the rotor disc is largely flowing downstream through the blades due to the blocking effect. As the tip release increases, a portion of the airflow flows downstream through the tip release, resulting in a change in pressure distribution and vortex distribution within the duct. As the blade tip clearance continues to increase, the duct gradually loses its grinding effect, at which point the tail rotor plays a single role. Consequently, the pressure and noise are much lower. The tip clearance, like the blade angle, must be selected within an appropriate range.

\subsection{Effect of lip radius change on tail rotor thrust and noise performance}

In this section, the effect of lip radius variation on thrust and noise is discussed. We discuss the effect of the radius of the inlet lip within the range of $120 \mathrm{~mm}$ to $142.5 \mathrm{~mm}$. The original design was built with a lip radius of $135 \mathrm{~mm}$. The overall sound pressure level and thrust calculation results for different inlet lip radii are shown in Table 7 . Figure 19 shows the directionality characteristics of farfield noise. 


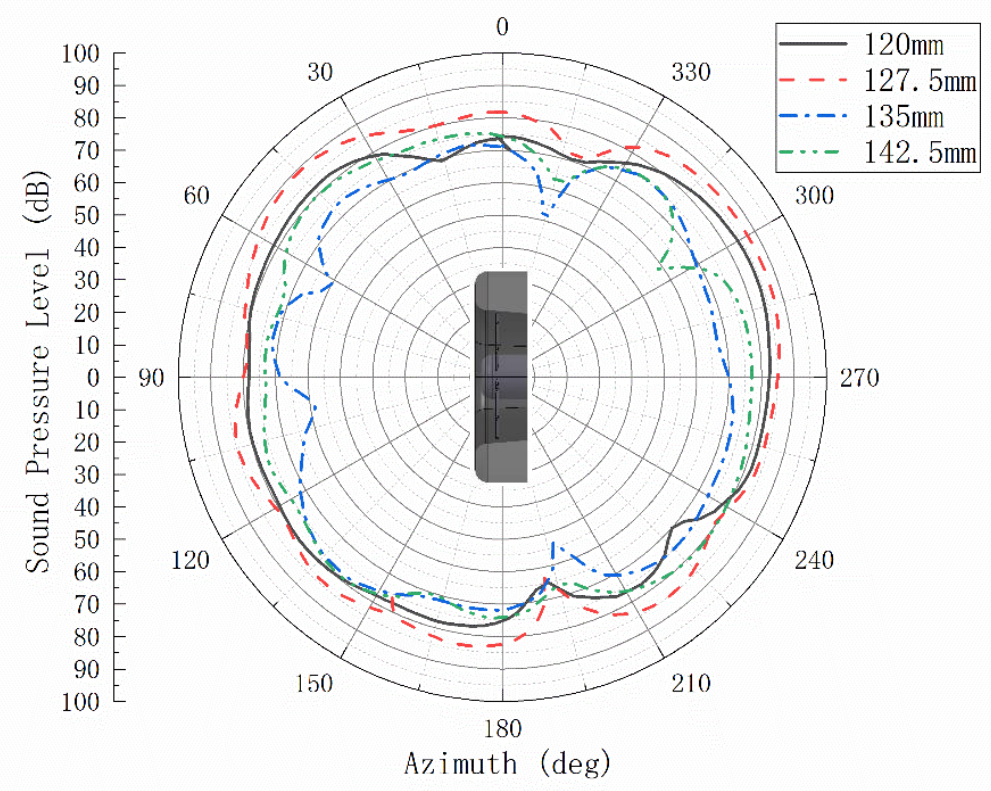

Figure 19. Far-field sound directional features of different lengths of lip radius.

Table 7. Lip radius change tasks and associate results.

\begin{tabular}{ccc}
\hline Lip radius & OASPL(dB) & Thrust(N) \\
\hline $120 \mathrm{~mm}$ & 78.1 & 9197.9 \\
$127.5 \mathrm{~mm}$ & 82.7 & 8476.2 \\
$135 \mathrm{~mm}$ & 70.2 & 7487.6 \\
$142.5 \mathrm{~mm}$ & 74.9 & 9418.4 \\
\hline
\end{tabular}

The overall sound pressure levels corresponding to the four lip radii were $78.1 \mathrm{dBA}, 82.7 \mathrm{dBA}$, $70.2 \mathrm{dBA}$, and $74.9 \mathrm{dBA}$, respectively, and the thrust showed some randomness with the change in lip radius. At the same time, the variation between thrust and sound due to the change in lip radius has a certain randomity. From the calculation results, when the lip radius is $127.5 \mathrm{~mm}$, the noise of the helicopter tail rotor is reduced by $15.1 \%$ and the thrust is increased by $13.2 \%$ compared with the original design.

\subsection{Comparison of the modification template with the original template}

In the preceding 6 sections, we analyzed the effects of structural parameters on the thrust and noise of the helicopter's tail rotor. Based on the results of the calculations, we proposed an enhanced design of the helicopter's tail rotor, taking into account the effect of each parameter on the noise and thrust of the helicopter's tail rotor. Table 8 shows the comparison of parameters between the original design and the upgraded design. Based on the calculation, we found that the enhanced tail rotor of the helicopter's duct had increased to $1242.9 \mathrm{~N}$ thrust compared to the original design. In the meantime, the sound of the helicopter's enhanced tail rotor duct decrease from $86.7 \mathrm{dBA}$ to $70.2 \mathrm{dBA}$.

Table 8. Specification modified in parameter between prototype and the new one.

\begin{tabular}{ccc}
\hline Specification & Origin design & Modified design \\
\hline Airfoil & NACA23012 & VR-12 \\
Chord length & $110 \mathrm{~mm}$ & $110 \mathrm{~mm}$ \\
Blade angle & $40 \mathrm{deg}$ & $43 \mathrm{deg}$ \\
Diffuser angle & $4 \mathrm{deg}$ & $4 \mathrm{deg}$ \\
Tip clearance & $11.25 \mathrm{~mm}$ & $18.75 \mathrm{~mm}$ \\
\hline
\end{tabular}




Lip radius $\quad 135 \mathrm{~mm} \quad 135 \mathrm{~mm}$

\section{Conclusions}

In this paper, a hybrid acoustic analogy analysis method is proposed and used to study the thrust and noise performance of a helicopter duct tail rotor. The flow field inside the duct is analyzed using the computational fluid dynamic method, and the far-field acoustic directional characteristics and overall sound pressure level of the duct tail rotor are analyzed using the acoustic finite element method.

The effects of blade airfoil, blade angle of attack, blade chord length, tip clearance, diffuser angle, and duct lip radius on the thrust and noise performance of the helicopter tail rotor are discussed separately. The results show that some parameters can positively affect the tail rotor thrust and noise, and some parameters need to be considered in the range of values in the structural design.

According to the calculations, and an improved reformed tail rotor was developed. By comparing with the original design, the overall sound pressure level of the helicopter tail rotor was reduced from $86.7 \mathrm{dBA}$ to $70.2 \mathrm{dBA}$, while the thrust was increased by $1242.9 \mathrm{~N}$. This digital method can help designers analyze the noise and thrust of the duct tail rotor during the design phase of the helicopter.

Author Contributions: conceptualization, Yuhong Jia and Aipeng Hao; methodology, Aipeng Hao; software, Aipeng Hao; validation, Aipeng Hao; formal analysis, Aipeng Hao.; writing - original draft preparation, Aipeng Hao.; writing - review and editing, Yuhong Jia; visualization, Aipeng Hao.

Conflicts of Interest: The authors declare no conflict of interest.

\section{References}

1. George, A.R. Helicopter noise: State-of-the-art. Journal of Aircraft 1978, 15, 707-715.

2. Chia, M.H.; Duraisamy, K.; Padthe, A.K.; Friedmann, P.P. Active and Passive Helicopter Noise Reduction Using the AVINOR/HELINOIR Code Suite. Journal of Aircraft 2017, 55, 727-740.

3. Piechowicz, R. Noise reducing profile for helicopter rotor blade tracking wedges. Google Patents: 2017.

4. Yang, T.; Brentner, K.S.; Corle, E.; Schmitz, S. Study of Active Rotor Control for In-Plane Rotor Noise Reduction. Journal of Aircraft 2018, 10.2514/1.C034873, 1-12, doi:10.2514/1.C034873.

5. Shi, Y.; Li, T.; He, X.; Dong, L.; Xu, G. Helicopter Rotor Thickness Noise Control Using Unsteady Force Excitation. Applied Sciences 2019, 9, 1351.

6. Yin, J.P.; Ahmed, S.R. Helicopter main-rotor/tail-rotor interaction. Journal of the American Helicopter Society 2000, 45, 293-302.

7. Cao, Y.; Yu, Z. Numerical simulation of turbulent flow around helicopter duct tail rotor. Aerospace Science and Technology 2005, 9, 300-306, doi:https://doi.org/10.1016/j.ast.2005.01.006.

8. Yin, J.P.; Ahmed, S.R. Aerodynamics and aeroacoustics of helicopter main-rotor/tail-rotor interaction. In Proceedings of Aeroacoustics Conference and Exhibit, AIAA/CEAS 1999, May 10, 1999 - May 12, 1999, Bellevue, WA, United states; pp. 834-844.

9. Filippone, A. Aircraft noise prediction. Progress in Aerospace Sciences 2014, 68, 27-63, doi:10.1016/j.paerosci.2014.02.001.

10. Brentner, K.S.; Farassat, F. Analytical comparison of the acoustic analogy and Kirchhoff formulation for moving surfaces. AIAA journal 1998, 36, 1379-1386.

11. Farassat, F. Derivation of Formulations 1 and 1A of Farassat. Nasa Tm 2007.

12. Schlegel, R.; King, R.; Mull, H. Helicopter rotor noise generation and propagation; UNITED TECHNOLOGIES CORP STRATFORD CT SIKORSKY AIRCRAFT DIV: 1966.

13. Lowson, M.; Ollerhead, J. A theoretical study of helicopter rotor noise. Journal of Sound and Vibration 1969, 9, 197-222.

14. Song, H.; Yi, M.; Huang, J.; Pan, Y.; Liu, D. Numerical method to compute acoustic diffuser effect of a moving source. SpringerPlus 2016, 5, 2117.

15. Yilmaz, S.; Erdem, D.; Kavsaoglu, M.S. Performance of a duct blade designed for UAV applications at zero angle of attack flight: An experimental study. Aerospace Science and Technology 2015, 45, 376-386, doi:https://doi.org/10.1016/j.ast.2015.06.005.

16. Karamolegkos, K. Duct Tail Rotor Perfomance Prediction Using CFD. 2014. 
17. Edwards, B.; Andrews, J.; Rahnke, C. Duct tail rotor designs for rotorcraft and their low noise features. In Proceedings of Proceedings of the FVP Symposium on "Advances in Rotorcraft Technology", Ottawa, Canada, AGARD-CP-592.

18. Roh, N.; Oh, S.; Park, D. Aerodynamic Characteristics of Helicopter with Duct Fan Tail Rotor in Hover under Low-Speed Crosswind. International Journal of Aerospace Engineering 2020, 2020, 7059209, doi:10.1155/2020/7059209.

19. Camussi, R.; Bennett, G.J. Aeroacoustics research in Europe: The CEAS-ASC report on 2019 highlights. Journal of Sound and Vibration 2020, 484, 115540, doi:https://doi.org/10.1016/j.jsv.2020.115540.

20. Farassat, F.; Brentner, K.S. The uses and abuses of the acoustic analogy in helicopter rotor noise prediction. Journal of the American Helicopter Society 1988, 33, 29-36.

21. Gabard, G.; Beriot, H.; Prinn, A.; Kucukcoskun, K. An Adaptive, High-Order Finite Element Method for Aeroengine Acoustics. In Proceedings of aiaa/ceas aeroacoustics conference.

22. Agrawal, B.R. Modeling fan broadband noise from jet engines and rod-airfoil benchmark case for broadband noise prediction. 2015.

23. Siozos-Rousoulis, L.; Lacor, C.; Ghorbaniasl, G. A flow control technique for noise reduction of a rodairfoil configuration. Journal of Fluids and Structures 2017, 69, 293-307, doi:10.1016/j.jfluidstructs.2017.01.003.

24. Bériot, H.; Modave, A. An automatic perfectly matched layer for acoustic finite element simulations in convex domains of general shape. International Journal for Numerical Methods in Engineering 2021, 122, 1239-1261, doi:https://doi.org/10.1002/nme.6560.

25. Gabard, G.; Bériot, H.; Prinn, A.G.; Kucukcoskun, K. Adaptive, High-Order Finite-Element Method for Convected Acoustics. AIAA Journal 2018, 56, 3179-3191, doi:10.2514/1.J057054.

(C) 2019 by the authors. Submitted for possible open access publication under the terms and conditions of the Creative Commons Attribution (CC BY) license (http://creativecommons.org/licenses/by/4.0/). 